\title{
Optimization of parasite DNA enrichment approaches to generate whole genome sequencing data for Plasmodium falciparum from low- parasitemia samples
}

\section{Zalak Shah}

University of Maryland School of Medicine

Matthew Adams

University of Maryland School of Medicine

Kara A Moser

University of Maryland School of Medicine

Biraj Shrestha

University of Maryland School of Medicine

Emily M Stucke

University of Maryland School of Medicine

Miriam K Laufer

University of Maryland School of Medicine

David Serre

University of Maryland School of Medicine

Joana C Silva

University of Maryland School of Medicine

Shannon Takala-Harrison ( $\nabla$ stakala@medicine.umaryland.edu )

University of Maryland School of Medicine https://orcid.org/0000-0003-4674-8500

\section{Methodology}

Keywords: Plasmodium falciparum, malaria, whole genome sequencing, selective whole genome amplification, vacuum filtration

Posted Date: February 18th, 2020

DOI: https://doi.org/10.21203/rs.2.17581/v2

License: (c) (i) This work is licensed under a Creative Commons Attribution 4.0 International License. Read Full License 
Version of Record: A version of this preprint was published at Malaria Journal on March 30th, 2020. See the published version at https://doi.org/10.1186/s12936-020-03195-8. 


\section{Abstract}

Background: Owing to the large amount of host DNA in clinical samples, generation of high-quality Plasmodium falciparum whole genome sequencing (WGS) data requires enrichment for parasite DNA. Enrichment is often achieved by leukocyte depletion of infected blood prior to storage. However, leukocyte depletion is difficult in low-resource settings and limits analysis to prospectively-collected samples. As a result, approaches such as selective whole genome amplification (sWGA) are being used to enrich for parasite DNA. However, sWGA has had limited success in generating reliable sequencing data from low parasitemia samples. In this study, we evaluated whether enzymatic digestion with MspJI prior to sWGA improved genome coverage compared to sWGA alone. We also examined the potential of sWGA to cause amplification bias in polyclonal infections. Methods: DNA extracted from lab-created dried blood spots was treated with a modification-dependent restriction endonuclease, MspJI, and filtered via vacuum filtration. Samples were then selectively amplified using a previously reported sWGA protocol and subjected to WGS. Genome coverage statistics were compared between the optimized sWGA approach and the previously reported sWGA approach performed in parallel. Differential amplification by sWGA was assessed by comparing WGS data generated from lab-created mixtures of parasite isolates, from the same geographical region, generated with or without sWGA. Results: MspJI digestion did not enrich for parasite DNA. Samples that underwent vacuum filtration (without MspJI digestion) prior to sWGA had the highest parasite DNA concentration and displayed greater genome coverage compared to MspJI+sWGA and sWGA alone, particularly for low parasitemia samples. The optimized sWGA (filtration + sWGA) approach was successfully used to generate WGS data from 218 non-leukocyte depleted field samples from Malawi. Sequences from lab-created mixtures of parasites did not show evidence of differential amplification of parasite strains compared to directly sequenced samples. Conclusion: This optimized sWGA approach is a reliable method to obtain WGS data from non-leukocyte depleted, low parasitemia samples. The absence of amplification bias in data generated from mixtures of isolates from the same geographic region suggests that this approach can be appropriately used for molecular epidemiological studies.

\section{Background}

Next-generation sequencing has greatly advanced research on malaria parasite genomics. Several molecular epidemiological studies have used genomic approaches in an effort to better understand Plasmodium falciparum genetic diversity in relation to malaria transmission, drug resistance and vaccine design [1-5]. However, a majority of such population genomics studies rely on whole genome sequencing of samples collected in malaria endemic areas. Since patient blood samples contain mostly human DNA, enrichment for parasite DNA is required in order to obtain parasite sequence data with adequate genome coverage. Leukocyte depletion is an effective method for reducing the amount of host DNA for parasite sequencing and hence increase the proportion of parasite DNA prior to sequencing; however, depletion must be performed within hours of sample collection and can be logistically challenging in some resource-limited settings $[6,7]$. In addition, once the sample is frozen and cells are lysed, leukocyte 
depletion is no longer effective, thus limiting the application of this approach to prospectively-collected samples. To reduce the need for extensive sample processing in the field and to enable examination of the wealth of historical samples collected as dried blood spots or whole venous blood, malaria researchers have explored alternative parasite DNA enrichment approaches including enzymatic digestion of human DNA [8], selective whole genome amplification (sWGA) [9], and hybrid selection (capture-based method) [10].

MspJI is a restriction endonuclease that cleaves specific motifs containing methylated cytosines that has been used to selectively digest human DNA prior to parasite DNA sequencing [11]. The success of this approach is based on the assumption of different methylation patterns in the human and parasite genomes; however, methylation patterns in $P$. falciparum are not fully understood [12]. sWGA of the parasite genome over the human genome has also shown promising results as a method for enrichment of parasite DNA prior to whole genome sequencing. This approach uses multiple displacement amplification with phi29 DNA polymerase using primers binding at greater density in the parasite genome compared to the human genome. Phi29 results in amplification of long DNA fragments and is known to have a low error rate. An existing sWGA protocol has been shown to work best with samples that have parasitemia greater than $\sim 1200$ parasites $/ \mu \mathrm{L}$ [9]. While this parasitemia threshold may allow sequencing of most clinical infections, it limits studies of lower parasitemia infections, including submicroscopic infections that may significantly contribute to the malaria burden in some areas [13]. In addition, because sWGA primers were designed against the 3D7 reference sequence without consideration of $P$. falciparum genetic diversity, the potential for differential amplification of particular parasite clones within a polyclonal infection (e.g. those most genetically similar to the reference) is a concern. Since a large proportion of infections from high-transmission areas are polyclonal, the possibility of amplification bias introduced by sWGA warrants further investigation, as such bias could lead to inaccurate inferences in downstream analyses.

In this study, we tested whether performing enzymatic digestion with MspJI prior to sWGA and whole genome sequencing improves genome coverage compared to sWGA alone when applied to samples representing a range of parasitemias. In addition, we also evaluated whether the optimized sWGA protocol results in biased estimates of multiplicity of infection or allele frequencies by comparing whole genome sequence data generated from lab-created mixtures of isolates from Malawi that underwent sWGA prior to sequencing or were directly sequenced.

\section{Methods}

\section{$\underline{\text { Lab-created samples to evaluate enrichment approaches }}$}

Dried blood spots. To test the different parasite DNA enrichment approaches, dried blood spots were created by mixing cultured NF54-infected red blood cells with uninfected whole human blood. The laboratory-adapted isolate, NF54, was maintained in culture following the method of Trager and Jensen [14]. Parasite concentrations were microscopically enumerated from sorbitol-synchronized ring-stage 
NF54 culture and mixed with uninfected whole human blood (Interstate Blood Bank, Nashville, TN) resulting in parasite concentrations ranging from 10,000 to 500 parasites $/ \mu \mathrm{L}$ and subsequently spotting $12.5 \mathrm{~mL}$ of blood onto Whatman $3 \mathrm{MM}$ filter paper. DNA was extracted from dried blood spots using the protocol described by Zainabadi et al[15]. The DNA was treated under three conditions, including sWGA only and MspJI or Msp $\mathrm{JI}^{-}$control (no enzyme)+ followed by sWGA, as illustrated in Supplementary Figure S1. The MpsJI- condition followed the same protocol as MspJI, without the enzyme.

Mixtures of DNA to assess amplification bias. DNA from four previously cultured and sequenced field isolates from Malawi [16] were mixed in equal proportions to assess potential amplification bias introduced by sWGA. The DNA concentration for each sample was measured using a picogreen assay (Thermo Fisher Scientific, Waltham, MA) and the samples were mixed in equal proportions. None of the cultured isolates represented polyclonal infections, based on analysis of prior sequencing data using estMOI [17]. The sample was further split into six tubes, three of which underwent direct whole genome sequencing and the other three which underwent sWGA, followed by whole genome sequencing, as shown in Figure 4.

Mspll Digestion. MspJI digestion was performed in a $0.2 \mathrm{~mL}$ 96-well PCR plate. The reaction mixture contained 1x CutSmart Buffer, $10 \mathrm{mg}$ of bovine serum albumin and 6 units of MspJI (New England Biolabs, Ipswich, MA). The MspJI digestion control (i.e., Msp $\mathrm{JI}^{-}$) contained the same buffers but excluded the enzyme. $25 \mathrm{~mL}$ of sample DNA was added to both reaction mixtures (total volume, $30 \mathrm{~mL}$ ), and reactions were incubated in a thermocycler. Two different incubation protocols were tested, one with a 16 hrs incubation at $37^{\circ} \mathrm{C}$, followed heating at $65^{\circ} \mathrm{C}$ for $20 \mathrm{~min}$ to inactivate the enzyme and cooling at $4^{\circ} \mathrm{C}$, and a second protocol where the $37^{\circ} \mathrm{C}$ incubation lasted only $4 \mathrm{hrs}$.

Vacuum filtration of DNA. Following enzymatic digestion, the entire reaction mixture was transferred to a MultiScreen ${ }^{\circledR}$ PCR Filter Plate (Millipore) and filtered to remove digested DNA fragments using a MultiScreen ${ }^{\circledR}$ Vacuum Manifold with a pressure of -7 inches $\mathrm{Hg}$ until the wells were emptied and the filters appeared dry. Filtered samples were reconstituted with $30 \mathrm{~mL}$ of water, and the plate was gently agitated for 15 mins. Samples were then transferred to a new plate.

sWGA. Amplification was performed in a $0.2 \mathrm{~mL}$ 96-well PCR plate. The reaction mixture contained $1 \mathrm{x}$ BSA, $1 \mathrm{mM}$ dNTPs, $2.5 \mathrm{mM}$ of each amplification primer, $1 \times$ Phi29 reaction buffer and 30 units of Phi29 polymerase. $17 \mathrm{~mL}$ of template DNA was added to the reaction mixture (total volume, $50 \mathrm{~mL}$ ) which was then placed in a thermocycler programmed for a stepdown protocol $\left(35^{\circ} \mathrm{C}\right.$ for $5 \mathrm{~min}, 34^{\circ} \mathrm{C}$ for $10 \mathrm{~min}$, $33^{\circ} \mathrm{C}$ for $15 \mathrm{~min}, 32^{\circ} \mathrm{C}$ for $20 \mathrm{~min}, 31^{\circ} \mathrm{C}$ for $30 \mathrm{~min}, 30^{\circ} \mathrm{C}$ for $16 \mathrm{hrs}$ ), followed by heating at $65^{\circ} \mathrm{C}$ to inactivate the enzyme and cooling at $4^{\circ} \mathrm{C}$. We used the same primers as Oyola et al [9] (see Supplementary Material).

qPCR. Quantitative PCR of the human actin gene and $P$. falciparum 18S rRNA gene was used to estimate the amount of human and parasite DNA, respectively, before and after sWGA. The reaction mixture contained QuantiTech 2x QT Multiplex Master Mix, $10 \mathrm{mM}$ of each of the primers and $1.5 \mathrm{~mL}$ of template 
DNA (total volume, $10 \mathrm{~mL}$ ). A two-tailed Mann-Whitney $U$ test was used to estimate differences between different experimental conditions (e.g. MspJI-sWGA, MspJI--sWGA, sWGA).

Whole genome sequencing. Genomic DNA libraries were constructed for sequencing using the KAPA Library Preparation Kit (Kapa Biosystems, Woburn, MA). DNA (500 hg) was fragmented with the Covaris E210 to 200 bp. Libraries were prepared using a modified version of the manufacturer's protocol. The DNA was purified between enzymatic reactions and library size selection was performed with AMPure XT beads. Libraries were assessed for concentration and fragment size using the DNA High Sensitivity Assay on the LabChip GX (Perkin Elmer, Waltham, MA). Library concentrations were also assessed by qPCR using the KAPA Library Quantification Kit. Libraries were pooled and sequenced on a 150 bp paired-end Illumina HiSeq 4000 run (Illumina, San Diego, CA).

\section{Data Analysis}

Read mapping and coverage. Each dataset was analyzed by mapping raw fastq files to the 3D7 reference genome using Bowtie2 [18]. Bam files were processed according to GATK's Best Practices workflow to obtain analysis-ready reads $[19,20]$. Bedtools [21] was used to generate coverage and depth estimates from the processed reads. Differences in the proportion of the genome covered in samples from untreated $v s$. filtered sWGA were tested using the z-score test for difference in proportions.

Variant calling. GATK's Best Practices workflow was followed for variant calling $[19,20]$. Haplotype Caller was used in reference confidence mode to create genomic variant call format (GVCF) files for each sample and joint SNP Calling (GATK v3.7). Variants were removed if they met the following filtering criteria: QD < 2.0, FS > 60.0, MQ < 40.0, MQRankSum <-12.5, ReadPosRankSum <-8.0, QUAL < 50. Variant sites with $>20 \%$ missing genotypes were additionally removed using vcftools.

Assessment of amplification bias. Whole genome sequence data generated from lab-created isolate mixtures that either underwent sWGA and sequencing or direct sequencing were compared to evaluate the potential for sWGA to introduce amplification bias. The experimental design is illustrated in Figure 4. Reference allele frequencies for each sample were estimated using samtools mpileup and were compared to examine the variability between and within samples from sWGA and non-sWGA groups. Sites with coverage depth lower than 20x were excluded from this analysis. In order to reduce coverage bias, we also eliminated sites that were not covered in all six samples. After applying these filters, $1,786,088$ sites remained. The distribution and spearman correlation coefficient (rho) were estimated in R.

$F_{W S}$ a measure of within-sample diversity, was used to estimate infection complexity for each isolate mixture for comparison between the sWGA and non-sWGA conditions. $F_{W S}$ was estimated using the R package, moimix (https://github.com/bahlolab/moimix). Significance was determined using the MannWhitney $\mathrm{U}$ test. Only the core $P$. falciparum genome was used to estimate $F_{\mathrm{WS}}$ [22].

The composition of each lab-created mixture was also compared to determine if there were significant differences between the sWGA and non-sWGA groups, implying differential amplification of certain 
strains over others. To assess potential amplification bias, we compared the proportion of isolate-specific SNPs for each isolate called from WGS data generated from isolate mixtures that did or did not undergo sWGA prior to sequencing. Isolate-specific SNPs were identified by comparing WGS data from each of the four isolates used to create the experimental mixtures. All variant sites with missing alleles in any of the isolates were removed from the analysis. For each isolate mixture, the predominant allele (defined as an allele comprising $>70 \%$ of reads) at each position was called; if no allele was predominant based on this threshold, the SNP was called missing. The positions of unique SNPs for each isolate were extracted from the sequence data generated from the isolate mixtures. The proportion of unique SNPs from each isolate in the mixture was estimated and compared between the sWGA and non-sWGA groups.

Significance was estimated using a z-score test for difference in proportions.

\section{Results}

Vacuum filtration, but not enzyme digestion, improves the quality of whole genome sequence data

Lab-created dried blood spots representing a range of parasitemias were created to test the different DNA enrichment approaches. DNA extracted from the dried blood spots underwent one of three conditions prior to sWGA: 1) MspJI digestion, 2) MspJI- control (same conditions as MspJl but without enzyme), or 3) untreated, as illustrated in Figure S1. Samples that underwent MspJI digestion had significantly less human ( $p=0.028$, Mann-Whitney $U$ test) and parasite DNA ( $p=0.028$, Mann-Whitney $U$ test) compared to the untreated samples. Similarly, MspJI-digested samples also had significantly less human ( $p=0.028$, Mann-Whitney $U$ test) and parasite DNA ( $p=0.028$, Mann-Whitney $U$ test) compared to the Msp $\mathrm{JI}^{-}$control (Figure 1A). This pattern was consistent following sWGA, with MspJI-sWGA samples having significantly less human DNA ( $p$-value $=0.028$, Mann-Whitney $U$ test) as well as parasite DNA ( $p$-value $=0.028$, MannWhitney U test) compared to the untreated-sWGA samples. Surprisingly, the Msp $\mathrm{JI}^{-}$control samples that underwent sWGA had a significantly higher parasite DNA concentration compared to samples digested with MspJI ( $p$-value $=0.028$, Mann-Whitney $U$ test) and untreated samples ( $p$-value $=0.028$, Mann-Whitney $U$ test) that underwent sWGA. Further experiments suggested that the vacuum filtration step in the Msp $\mathrm{JI}^{-}$condition (Figure S1) was likely responsible for the improved parasite DNA concentration (Figure 1B, $p$-value $=0.028$, Mann-Whitney $U$ test), possibly due to removal of small DNA fragments that may lead to non-specific binding. This result was also consistent across samples with lower parasitemias, ranging from 5000 parasites $/ \mu \mathrm{L}$ to 500 parasites/ $\mu \mathrm{L}$ (Figure S2, p-value=0.028, Mann-Whitney U test).

The untreated-sWGA (no treatment, only sWGA) and filtered-sWGA samples (vacuum filtration, followed by sWGA), with different parasitemias $(10,000,1000,500$ parasites $/ \mu \mathrm{L})$, underwent whole genome sequencing, and the sequence reads were trimmed and mapped to the $P$. falciparum 3D7 reference genome. As seen in Figure 2A, the filtered-sWGA samples had a higher percentage of reads map to 3D7 ( $p$ $<0.0001, \mathrm{z}$ score test for difference in proportions). This pattern was more notable for samples with lower parasitemia (Figure 2A). Sequence data from filtered-sWGA samples displayed a slightly higher percent of the genome with $5 x$ coverage per million reads sequenced compared to sequence data from untreated- 
sWGA samples (Figure 2B). Further in-depth coverage analysis showed that this pattern was consistent across all chromosomes (Figure S3A). Visual inspection of coverage along chromosome 1 showed uneven coverage in both filtered and unfiltered samples that underwent sWGA, but filtered-sWGA samples had higher coverage in most regions, particularly in the lowest parasitemia sample. Indeed, in the lowest parasitemia sample, we observe higher coverage in several regions where the untreated-sWGA sample had very little or no coverage (Figure S3B).

We next tested the optimized sWGA protocol on 218 red blood cell pellets from Malawi that were PCRpositive for $P$. falciparum and had parasitemias, ranging from 0 to $>200,000$ parasites $/ \mu \mathrm{L}$ (by microscopy). Only $23 / 218$ (10.55\%) samples had less than $75 \%$ of the genome with $>=5 x$ coverage. Samples with $>75 \%$ of the genome with $>=5 x$ coverage had a median average read depth of $\sim 137 x$. Out of 50 samples with parasitemia $<500$ parasites $/ \mathrm{mL}$, only $9(18 \%)$ samples had less than $75 \%$ of the genome with $>=5 x$ coverage, while the remaining samples with $>75 \%$ of the genome with $>=5 x$ coverage had a median average read depth of $\sim 129 x$. We also estimated the correlation between percent genome coverage and parasitemia (Figure 3 ) and found a weak, but statistically significant, correlation between parasitemia and percent genome coverage $(p=0.00012$, Spearman's correlation rho $=0.25)$.

Optimized sWGA does not show evidence of amplification bias when applied to mixtures of parasite isolates from the same geographic region.

To examine the potential for amplification bias introduced by sWGA, we created equal mixtures of four $P$. falciparum isolates from Malawi. Three aliquots of these mixtures underwent sWGA followed by whole genome sequencing and three underwent whole genome sequencing without prior sWGA (Figure 4). Mixtures that underwent sWGA had a larger proportion of sequenced reads that mapped to the 3D7 reference genome compared to directly-sequenced mixtures, while directly-sequenced mixtures had a higher percentage of the genome with at least $5 x$ coverage (Table 1 ).

Table 1. Sequencing statistics in samples that underwent direct sequencing versus sWGA.

\begin{tabular}{|l|l|l|}
\hline & Direct Sequencing $(\mathrm{n}=3)$ & sWGA $(\mathrm{n}=3)$ \\
\hline Total reads sequenced & $30,878,334$ & $33,586,527$ \\
\hline Reads mapped to $P$. falciparum $(\%)$ & 92.21 & 95.52 \\
\hline Genome with 5x coverage $(\%)$ & 98.68 & 95.53 \\
\hline Mean coverage depth & $196 \mathrm{x}$ & $213 \mathrm{x}$ \\
\hline Total SNPs & 32,775 & 22,355 \\
\hline$F_{\text {WS }}$ & $0.068 \pm 0.009$ & $0.116 \pm 0.015$ \\
\hline
\end{tabular}

Three approaches were used to evaluate the potential for amplification bias by sWGA (Figure 4). First, we estimated the correlation between reference allele frequencies for each variant site in sequence data generated from mixtures that did or did not undergo sWGA prior to sequencing. Based on visual examination, the distribution of reference allele frequencies was similar in all six samples, but directly- 
sequenced samples had more clearly distinct peaks representing each of the four isolates compared to samples that underwent sWGA prior to sequencing (Figure S4). The correlation between reference allele frequencies was high (>94\%) and similar within and between sWGA or directly-sequenced samples (Figure S5).

Second, we estimated $F_{\mathrm{ws}}$ a measure of within-sample diversity [2,23,24], to examine differences in infection complexity between samples that underwent sWGA prior to sequencing and those that did not. $F_{\text {ws }}$ values range from 0 to 1 , with 0 indicating a mixture of highly unrelated clones and 1 indicating a single clone. Directly-sequenced samples had lower average $F_{\text {ws }}$ estimates than samples that underwent sWGA (Table 1). However, when $F_{\text {ws }}$ was estimated based on the subset of SNPs called in both groups, there was no significant difference in $F_{\text {ws }}$ between groups ( $p$-value=0.1, Mann Whitney U Test).

Finally, we compared the proportion of each isolate in our isolate mixtures based on the frequency of isolate-specific variants in sequence data generated from the mixtures that did or did not undergo sWGA. The proportion of isolate-specific SNPs in each mixture did not differ significantly between mixtures that underwent sWGA and those that did not (Figure 5).

\section{Discussion}

While advances in next-generation sequencing technologies have greatly expanded research in the field of malaria genomics, the difficulties of enriching for malaria parasite DNA in clinical samples, particularly those collected from submicroscopic infections, has limited population genomics analyses to include mostly high-parasitemia, symptomatic infections. In this study, we evaluated whether combining two published parasite DNA enrichment approaches, namely enzyme digestion of human DNA [8] and sWGA [9], prior to whole genome sequencing improved our ability to generate high-quality whole genome sequence data from non-leukocyte-depleted clinical samples with low parasitemia. Oyola et al. reported up to $\sim 9$ fold $P$. falciparum DNA enrichment resulting in $>98 \%$ of the parasite genome with at least $5 \mathrm{x}$ coverage when using MspJI digestion only (no sWGA) prior to sequencing [8]. However, we observed that enzyme digestion with MspJI resulted in a significant decrease in both human and parasite DNA concentrations, before and after sWGA. This reduced DNA concentration may be due to digestion of both human and parasite DNA, consistent with the presence of a "smear" of small DNA fragments observed when digested samples were subjected to gel electrophoresis (data not shown). This finding contrasts with that of Oyola et al. who observed a band representing intact parasite DNA along with digested human DNA following MspJI digestion, although it is notable that the amount of starting DNA (1ug total) used in the Oyola study was much larger than that obtained from the dried blood spots in our study, and likely larger than what would be obtained from dried blood spots collected in the field. In addition, Cowell et al. [24] observed no significant difference in genome coverage with digestion using MspJI or FspEI prior to sWGA and sequencing of $P$. vivax. However, since MspJl targets specific motifs containing methylated cytosines, and $P$. vivax has higher GC content than $P$. falciparum, it is possible that the targeted motifs may be more common in $P$. vivax, leading to the failure of MspJI in this context. Though 
we did not have success with MspJl, further investigation is warranted to identify alternative enzymes that target the human genome over the parasite genome that could potentially perform better and be useful in combination with sWGA.

While we did not find that digestion with MspJl improved parasite DNA concentration, to our surprise, we found that parasite DNA concentration was significantly greater in the Msp $\mathrm{JI}^{-}$control compared to Msp I and no treatment. Subsequent experiments suggested that this increased DNA concentration resulted from the vacuum filtration step. Indeed, we found that filtration of extracted DNA prior to sWGA resulted in a greater parasite DNA concentration compared to unfiltered DNA that underwent sWGA. We hypothesize that this result may be due to removal of small fragments of parasite DNA that may bind sWGA primers but not lead to effective amplification because of their short length. The increased DNA concentration obtained from the optimized sWGA protocol also resulted in increased genome coverage of whole genome sequencing data, although this increase was most pronounced in lower parasitemia samples.

Using the optimized sWGA approach, we were able to obtain high genome coverage from DNA extracted from lab-created dried blood spots with parasitemias as low as 500 parasites $/ \mu \mathrm{L}$, as well as from nonleukocyte depleted red blood cell pellets from the field, including 41 samples from low parasitemia, submicroscopic infections. Although statistically significant, the correlation between parasitemia and percent of the genome with $5 x$ coverage was small, suggesting that this optimized sWGA approach can be used to obtain high-quality whole genome sequence data from low parasitemia samples. Additional testing of this approach on dried blood spots stored under different environmental conditions and storage times will be required to further characterize which samples are likely to yield successful results using this optimized approach. If the parasite DNA is highly degraded, this optimized approach may not be successful, both because small DNA fragments may be removed during filtration and lead to less effective whole genome amplification and because of a general lack of longer fragments needed for successful sWGA. For more highly degraded samples, capture-based methods of enrichment may yield better results.

Amplification bias following sWGA or capture-based parasite DNA enrichment has been largely understudied but is essential to understand given the high prevalence of polyclonal infections in high transmission areas. We further explored the potential for amplification bias following sWGA by comparing whole genome sequence data generated from experimental mixtures of parasite DNA from four culture-adapted parasite isolates from Malawi that were either directly sequenced or underwent sWGA prior to sequencing. Consistent with other studies, genome coverage of sequence data generated from samples that underwent sWGA was more uneven compared to coverage of sequence data generated through direct sequencing, most likely due to the sparse sWGA primer coverage in diverse and AT-rich subtelomeric regions $[9,25]$. However, pairwise per base reference allele frequencies within the core genome were highly correlated both within and between the sWGA and directly-sequenced groups, suggesting that sWGA is not substantially biasing allele frequencies. Experimental mixtures that underwent sWGA had significantly higher estimates of $F_{\mathrm{ws}}$ implying some loss of within-mixture 
diversity. This result is in agreement with the results of Cowell et al. who found reduced estimates of infection complexity following sWGA of $P$. vivax DNA, based on analysis of both sequence data and microsatellites [24], and may be explained by differences in genome coverage between groups. Indeed, directly sequenced samples had higher genome coverage than samples that underwent sWGA, even in the core genome. To test this hypothesis, we estimated $F_{\text {WS }}$ based only on SNPs called in both the sWGA samples and the directly sequenced samples and observed no significant difference in $F_{\text {WS }}$ between groups, suggesting the lower diversity in samples that underwent sWGA was not the result of amplification bias favoring some isolates over others. This conclusion is also supported by the lack of significant differences in the proportion of isolate-specific variants between the sWGA and directly sequenced isolates. More in-depth analysis will be required to evaluate which genomic regions have reduced coverage in sWGA samples and the implications for downstream analyses.

While we did not observe preferential amplification based on equal mixtures of isolates from Malawi based on variants called against the 3D7 reference (believed to be of African origin $[16,26]$ ), it would be informative to evaluate this phenomenon in mixtures of parasites from other geographic regions or in varying proportions to determine whether one set of sWGA primers will provide unbiased results and high genome coverage in all settings or whether sWGA primers designed based on regional reference genomes is necessary. Because parasites from different continents are more genetically differentiated than parasites from the same geographic region [2], amplification bias may be more of a concern for comparisons involving parasites sampled from different continents than for comparisons of parasites from the same geographic region.

\section{Conclusions}

The optimized sWGA approach is a reliable method to obtain WGS data from non-leukocyte depleted, low parasitemia samples. The absence of amplification bias in data generated from mixtures of isolates from the same geographic region suggests that this approach can be appropriately used for molecular epidemiological studies.

\section{List Of Abbreviations}

DBS: dried blood spots

MOI: multiplicity of infection

sWGA: selective whole genome amplification

WGS: whole genome sequencing

\section{Declarations}

Ethics approval and consent to participate 
Red blood cell pellets used in this study were collected as part of the Mfera Cohort Study conducted in Malawi from 2014-2017 [27]. Written informed consent was obtained from parents or guardians of all study participants according to protocols approved by institutional review boards at the University of Maryland School of Medicine and the National Health Sciences Research Committee of Malawi.

\section{Consent for publication}

Not applicable.

Availability of data and materials

The datasets used and/or analyzed during the current study are available from the corresponding author on reasonable request.

\section{Competing interests}

The authors declare that they have no competing interests.

\section{Funding}

This work was supported by funding from the following awards granted by the National Institutes of Health: R01Al101713, R01Al125579, U19Al110820, K24Al114996, and the Malawi International Center of Excellence for Malaria Research U19AI089683.

\section{Authors' contributions}

ZS, MA, DS, JCS and ST-H designed the experiments. MKL provided samples. ZS and BS carried out the experiments. ZS, KM and EMS performed data analysis. MA, MKL, DS, JCS and ST-H provided guidance on data analysis. ZS and ST-H drafted the manuscript, and MA, KM, BS, EMS, MKL, DS and JCS approved the manuscript for publication.

\section{Acknowledgements}

We thank the participants in the Mfera Cohort Study. We would also like to thank Sudhaunshu Joshi and Gillian Mbambo for their assistance in culturing parasites and other lab experiments. We would also like to acknowledge Karl Seydel, Amed Ouattara, and Andrea Buchwald for their valuable input and suggestions, and Don Mathanga and Terrie Taylor for their role in leading the Malawi International Center of Excellence for Malaria Research.

\section{References}

1. Auburn S, Barry AE. Dissecting malaria biology and epidemiology using population genetics and genomics. Int J Parasitol. 2017 Feb 1;47(2):77-85. 
2. Manske M, Miotto O, Campino S, Auburn S, Almagro-Garcia J, Maslen G, et al. Analysis of Plasmodium falciparum diversity in natural infections by deep sequencing. Nature. 2012 Jul;487(7407):375-9.

3. Agrawal S, Moser KA, Morton L, Cummings MP, Parihar A, Dwivedi A, et al. Association of a Novel Mutation in the Plasmodium falciparum Chloroquine Resistance Transporter With Decreased Piperaquine Sensitivity. J Infect Dis. 2017 Aug 15;216(4):468-76.

4. Takala SL, Coulibaly D, Thera MA, Batchelor AH, Cummings MP, Escalante AA, et al. Extreme Polymorphism in a Vaccine Antigen and Risk of Clinical Malaria: Implications for Vaccine Development. Sci Transl Med. 2009 Oct 14;1(2):2ra5-2ra5.

5. Dwivedi A, Reynes C, Kuehn A, Roche DB, Khim N, Hebrard M, et al. Functional analysis of Plasmodium falciparum subpopulations associated with artemisinin resistance in Cambodia. Malar J. 2017 Dec 19;16(1):493.

6. Venkatesan M, Amaratunga C, Campino S, Auburn S, Koch O, Lim P, et al. Using CF11 cellulose columns to inexpensively and effectively remove human DNA from Plasmodium falciparum-infected whole blood samples. Malar J. 2012 Feb 10;11:41.

7. Auburn S, Campino S, Clark TG, Djimde AA, Zongo I, Pinches R, et al. An effective method to purify Plasmodium falciparum DNA directly from clinical blood samples for whole genome high-throughput sequencing. PloS One. 2011;6(7):e22213.

8. Oyola SO, Gu Y, Manske M, Otto TD, O’Brien J, Alcock D, et al. Efficient Depletion of Host DNA Contamination in Malaria Clinical Sequencing. J Clin Microbiol. 2013 Mar 1;51(3):745-51.

9. Oyola SO, Ariani CV, Hamilton WL, Kekre M, Amenga-Etego LN, Ghansah A, et al. Whole genome sequencing of Plasmodium falciparum from dried blood spots using selective whole genome amplification. Malar J [Internet]. 2016 Dec 20 [cited 2018 Mar 28];15. Available from: https://www.ncbi.nlm.nih.gov/pmc/articles/PMC5175302/

10. Melnikov A, Galinsky K, Rogov P, Fennell T, Van Tyne D, Russ C, et al. Hybrid selection for sequencing pathogen genomes from clinical samples. Genome Biol. 2011;12(8):R73.

11. Cohen-Karni D, Xu D, Apone L, Fomenkov A, Sun Z, Davis PJ, et al. The MspJI family of modificationdependent restriction endonucleases for epigenetic studies. Proc Natl Acad Sci U S A. 2011 Jul 5;108(27):11040-5.

12. Baumgarten S, Bryant JM, Sinha A, Reyser T, Preiser PR, Dedon PC, et al. Transcriptome-wide dynamics of extensive m6A mRNA methylation during Plasmodium falciparum blood-stage development. Nat Microbiol. 2019;4(12):2246-59.

13. Bousema T, Okell L, Felger I, Drakeley C. Asymptomatic malaria infections: detectability, transmissibility and public health relevance. Nat Rev Microbiol. 2014 Dec;12(12):833-40.

14. Trager W, Jensen JB. Human malaria parasites in continuous culture. 1976. J Parasitol. 2005 Jun;91(3):484-6.

15. Zainabadi K, Adams M, Han ZY, Lwin HW, Han KT, Ouattara A, et al. A novel method for extracting nucleic acids from dried blood spots for ultrasensitive detection of low-density Plasmodium 
falciparum and Plasmodium vivaxinfections. Malar J. 2017 Sep 18;16(1):377.

16. Moser KA, Drábek EF, Dwivedi A, Stucke EM, Crabtree J, Dara A, et al. Strains used in whole organism Plasmodium falciparum vaccine trials differ in genome structure, sequence, and immunogenic potential. Genome Med. 2020 Jan 8;12(1):6.

17. Assefa SA, Preston MD, Campino S, Ocholla H, Sutherland CJ, Clark TG. estMOI: estimating multiplicity of infection using parasite deep sequencing data. Bioinforma Oxf Engl. 2014 May $1 ; 30(9): 1292-4$.

18. Langmead B, Salzberg SL. Fast gapped-read alignment with Bowtie 2. Nat Methods. 2012 Apr;9(4):357-9.

19. DePristo MA, Banks E, Poplin R, Garimella KV, Maguire JR, Hartl C, et al. A framework for variation discovery and genotyping using next-generation DNA sequencing data. Nat Genet. 2011 May;43(5):491-8.

20. Van der Auwera GA, Carneiro MO, Hartl C, Poplin R, Del Angel G, Levy-Moonshine A, et al. From FastQ data to high confidence variant calls: the Genome Analysis Toolkit best practices pipeline. Curr Protoc Bioinforma. 2013;43:11.10.1-33.

21. Quinlan AR, Hall IM. BEDTools: a flexible suite of utilities for comparing genomic features. Bioinformatics. 2010 Mar 15;26(6):841-2.

22. Miles A, lqbal Z, Vauterin P, Pearson R, Campino S, Theron M, et al. Indels, structural variation, and recombination drive genomic diversity in Plasmodium falciparum. Genome Res. 2016 Sep;26(9):1288-99.

23. Chan ER, Menard D, David PH, Ratsimbasoa A, Kim S, Chim P, et al. Whole Genome Sequencing of Field Isolates Provides Robust Characterization of Genetic Diversity in Plasmodium vivax. PLoS Negl Trop Dis [Internet]. 2012 Sep 6 [cited 2019 Jul 22];6(9). Available from:

https://www.ncbi.nlm.nih.gov/pmc/articles/PMC3435244/

24. Cowell AN, Loy DE, Sundararaman SA, Valdivia H, Fisch K, Lescano AG, et al. Selective WholeGenome Amplification Is a Robust Method That Enables Scalable Whole-Genome Sequencing of Plasmodium vivax from Unprocessed Clinical Samples. mBio [Internet]. 2017 Feb 7 [cited 2019 Mar 24];8(1). Available from: https://www.ncbi.nlm.nih.gov/pmc/articles/PMC5296604/

25. Sundararaman SA, Plenderleith LJ, Liu W, Loy DE, Learn GH, Li Y, et al. Genomes of cryptic chimpanzee Plasmodium species reveal key evolutionary events leading to human malaria. Nat Commun. 2016 Mar 22;7:11078.

26. Preston MD, Campino S, Assefa SA, Echeverry DF, Ocholla H, Amambua-Ngwa A, et al. A barcode of organellar genome polymorphisms identifies the geographic origin of Plasmodium falciparum strains. Nat Commun. 2014 Jun 13;5:4052.

27. Buchwald AG, Sixpence A, Chimenya M, Damson M, Sorkin JD, Wilson ML, et al. Clinical Implications of Asymptomatic Plasmodium falciparum Infections in Malawi. Clin Infect Dis Off Publ Infect Dis Soc Am. 2019 01;68(1):106-12. 


\section{Additional File}

Additional file 1:

File name: AdditionalFile1.pdf

Title: AdditionalFile1

Description: Supplementary Material.

\section{Figures}
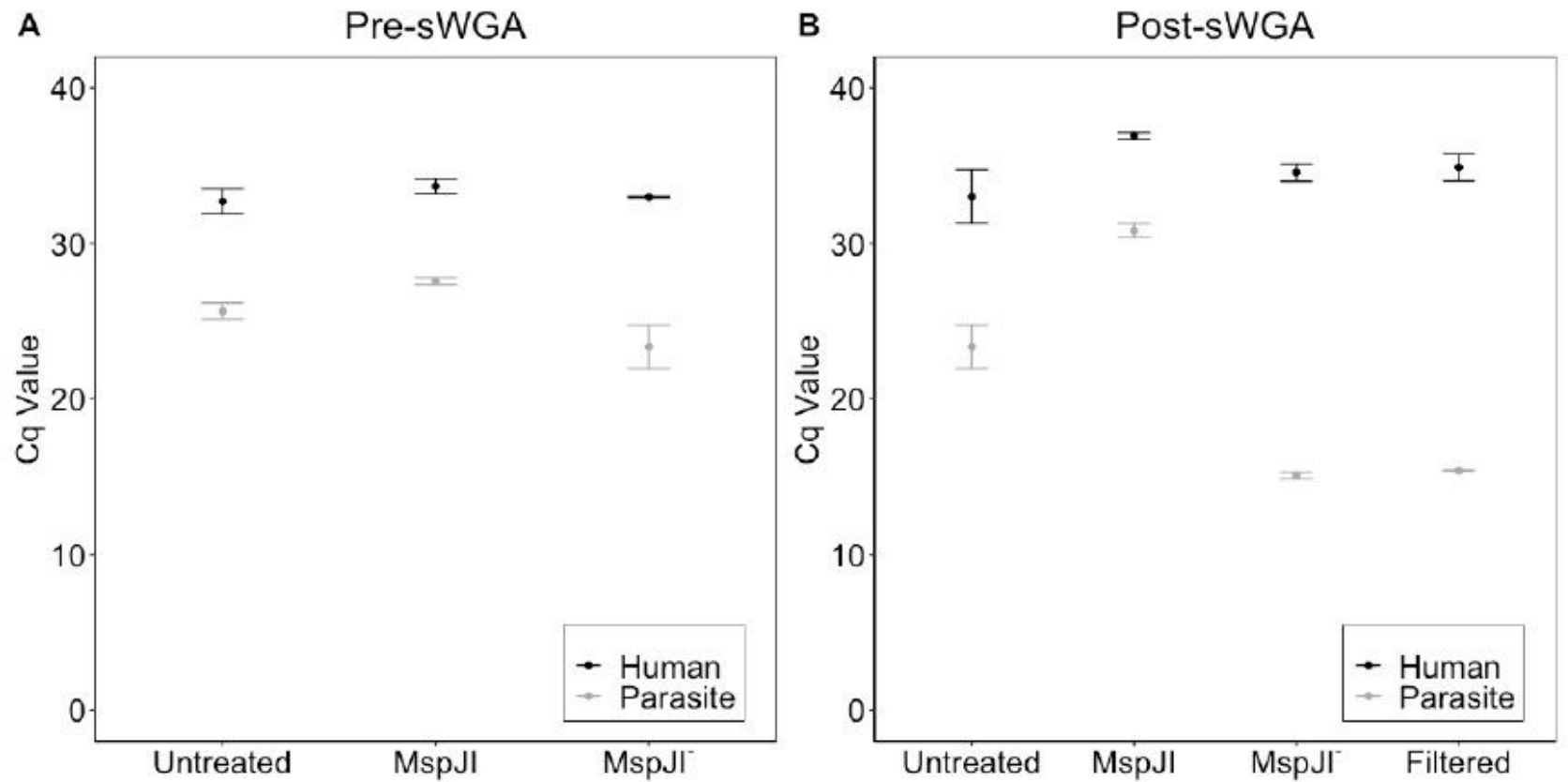

Figure 1

\section{Figure 1}

Effect of MspJI and sWGA treatments on parasite DNA concentration. (A) Human and P. falciparum Cq values prior to sWGA on samples with 10,000 parasites/ $\mathrm{LL}(n=3)$. Cq value indicates the number of cycles required to detect a signal, where higher Cq values indicate lower DNA concentrations. (B) Human and P. falciparum Cq values after sWGA on samples with 10,000 parasites/ $\square \mathrm{L}(\mathrm{n}=3)$. 

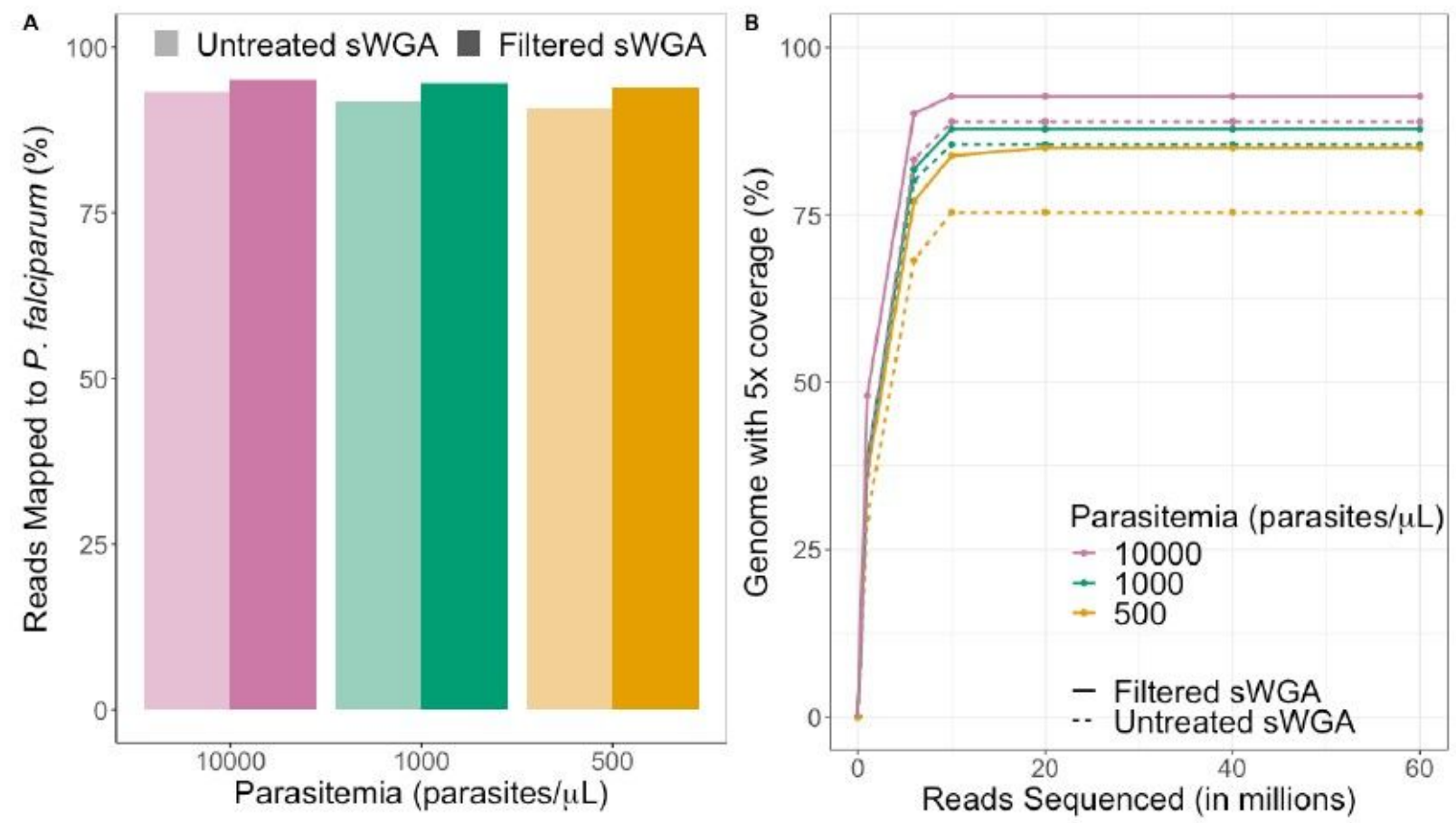

Figure 2

\section{Figure 2}

P. falciparum genome coverage in filtered and untreated samples that underwent 424 sWGA. (A) The percentage of reads that mapped to the P. falciparum 3D7 reference are shown 425 for filtered and untreated samples with different parasitemias that underwent sWGA prior to 426 sequencing. (B) Percentage of the P. falciparum 3D 7 genome with at least $5 x$ coverage is shown 427 relative to the number of reads sequenced (in millions) in filtered and untreated samples of 428 different parasitemias that underwent sWGA prior to sequencing. 


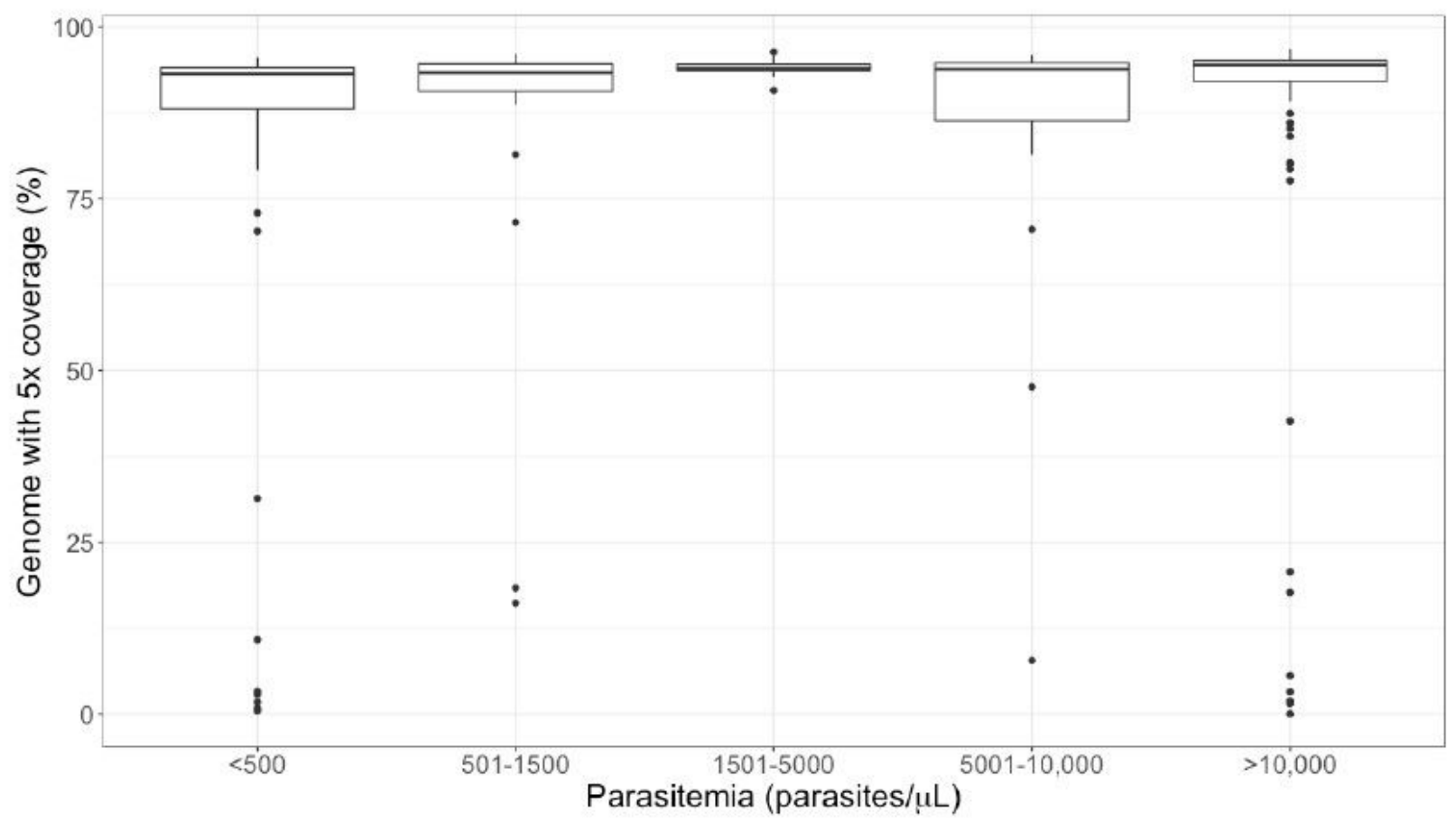

Figure 3

\section{Figure 3}

Percent of genome with $5 x$ coverage in whole genome sequences of field isolates 431 with different parasitemias.

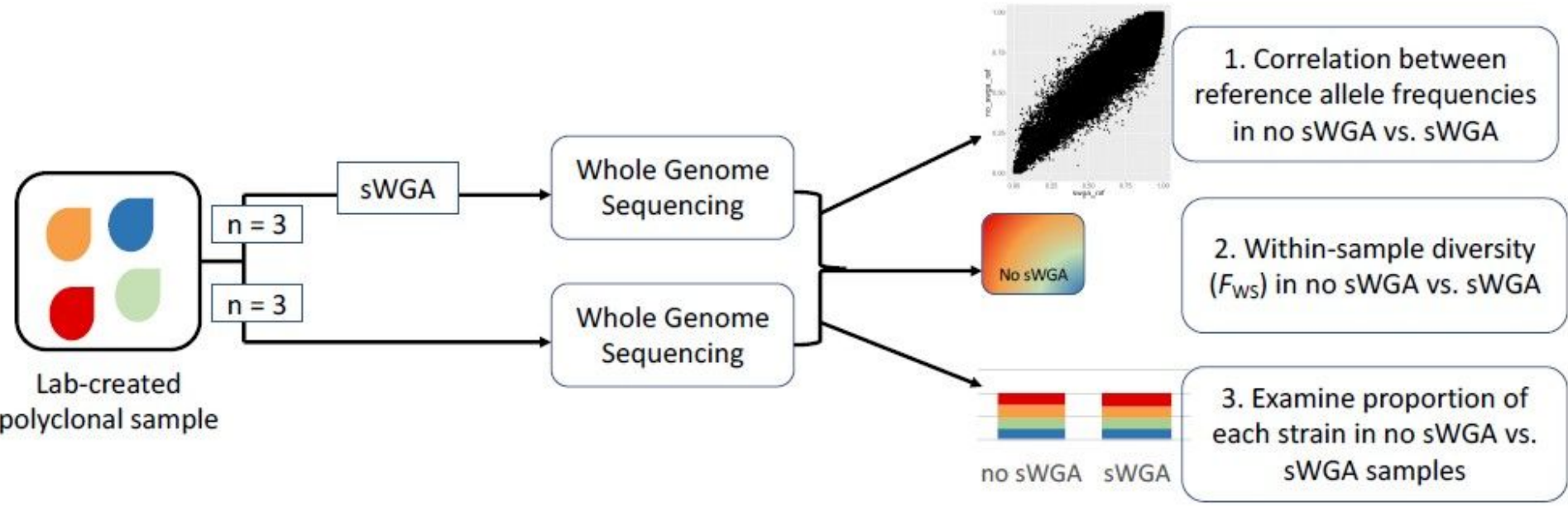

Figure 4 
Figure 4

Schematic of experimental design to evaluate potential amplification bias in samples 434 undergoing sWGA.

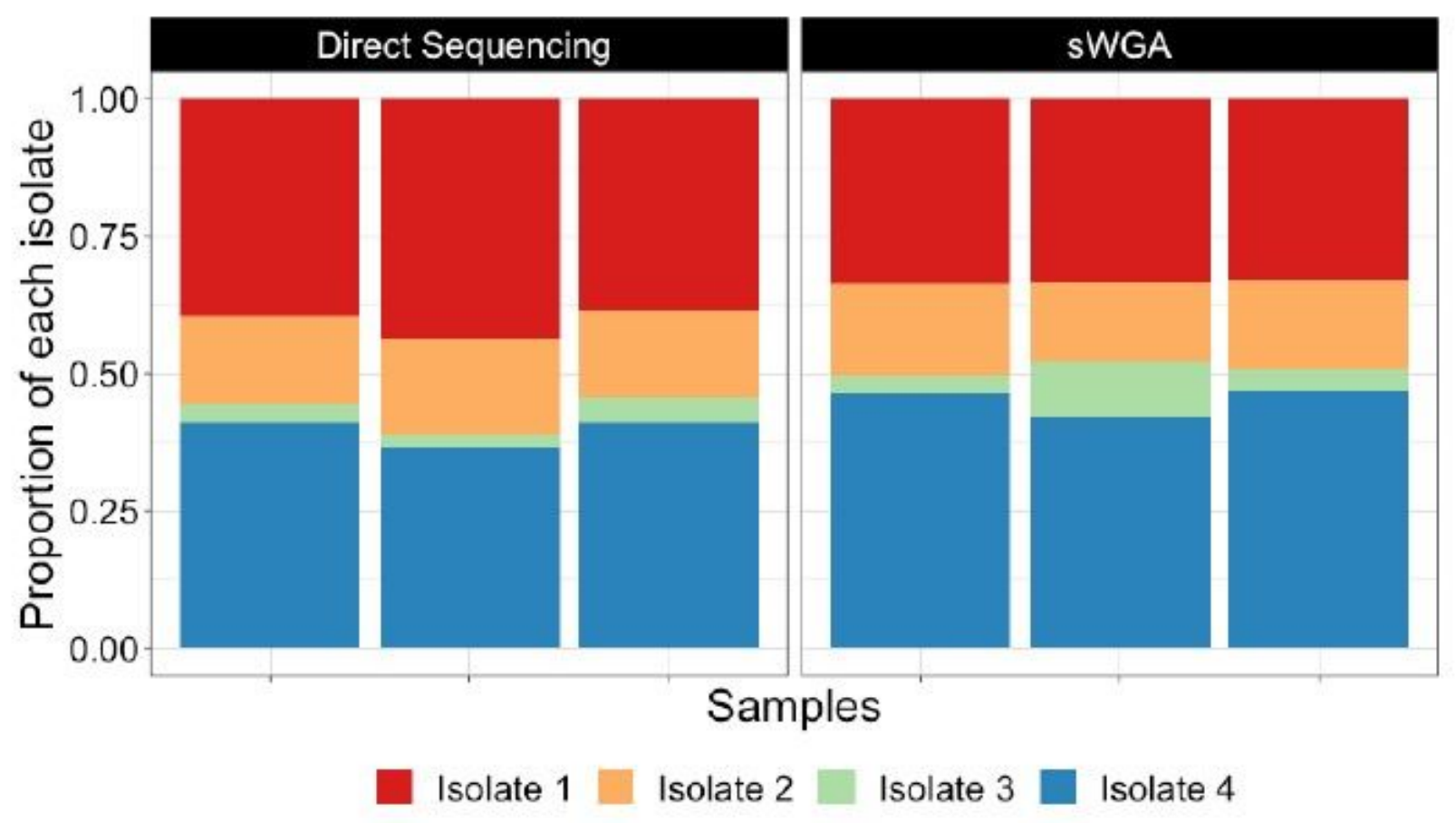

Figure 5

\section{Figure 5}

Proportion of isolate-specific SNPs in mixtures that underwent sWGA prior to 437 sequencing or were directly sequenced.

\section{Supplementary Files}

This is a list of supplementary files associated with this preprint. Click to download.

- AdditionalFilerevision1final.pdf 